\title{
Correction to: Mortality burden associated with all-cause pneumonia among adults with autoimmune inflammatory rheumatic diseases, human immunodeficiency virus infection, and malignancies: a population-based comparative study for informed decision-making in public health policies
}

\author{
Rodrigo Poubel Vieira de Rezende ${ }^{1}$ - Evandro Mendes Klumb ${ }^{2} \cdot$ Gecilmara Salviato Pileggi $^{3}$ \\ Published online: 21 July 2020 \\ (C) International League of Associations for Rheumatology (ILAR) 2020
}

Correction to: Clinical Rheumatology (2020) 39:1703-1705

https://doi.org/10.1007/s10067-020-05039-x

Table 2 in the original version of the above article was incorrectly presented. The data were misaligned during publication process. The correct Table 2 is shown as follows:

The online version of the original article can be found at https://doi.org/ 10.1007/s10067-020-05039-x

Rodrigo Poubel Vieira de Rezende ropoubel@id.uff.br

1 Departamento de Medicina Clínica, Hospital Universitário Antônio Pedro (HUAP), Universidade Federal Fluminense, Av. Marquês do Paraná, 303, Centro, Niterói, RJ 24030-210, Brazil

2 Departamento de Reumatologia, Universidade do Estado do Rio de Janeiro, Rio de Janeiro, Brazil

3 Faculdade de Ciências da Saúde de Barretos (FACISB), Barretos, SP, Brazil 
Table 2 Comparison of the frequency of mortality associated with allcause pneumonia between adults aged $\geq 19$ years with autoimmune inflammatory rheumatic diseases, human immunodeficiency virus infection, and malignant neoplasms. Mortality data from the general population of the State of Rio de Janeiro, Brazil, 2006-2018

PMN-associated deaths, $n$

non-PMN-associated deaths, $n$

Odds ratio $(95 \% \mathrm{CI})^{\mathrm{a}}$

$P$ value (AIRD group / comparator group) (AIRD group / comparator group)

Disease groups, women

CTD vs HIV
CTD vs malignant neoplasms
RA vs HIV

RA vs malignant neoplasms

Disease groups, men

$\begin{array}{lc}496 / 2227 & 1,250 / 5631 \\ 496 / 11,313 & 1,250 / 117,308 \\ 215 / 2227 & 261 / 5631 \\ 215 / 11,313 & 261 / 117,308 \\ & \\ 102 / 3753 & 259 / 9858 \\ 102 / 13,812 & 259 / 114,236 \\ 58 / 3753 & 56 / 9858 \\ 58 / 13,812 & 56 / 114,236\end{array}$

$1.0(0.89-1.12)$ 0.95

$4.1(3.70-4.57)$ $<0.0001$ $<0.0001$ $<0.0001$

CTD vs HIV

CTD vs malignant neoplasms

$58 / 13,812$

RA vs malignant neoplasms

$56 / 114,236$

\begin{tabular}{lc}
$1.0(0.82-1.30)$ & 0.77 \\
$3.3(2.58-4.09)$ & $<0.0001$ \\
$2.7(1.88-3.93)$ & $<0.0001$ \\
$8.6(5.93-12.37)$ & $<0.0001$ \\
\hline
\end{tabular}

${ }^{\text {a }}$ For between-group comparisons, the odds ratio formula is given by: (PMN-associated deaths in the CTD or RA group $\times$ non-PMN-associated deaths in the $\mathrm{HIV}$ or malignant neoplasm group) $\div$ (non-PMN-associated deaths in the CTD or RA group $\times \mathrm{PMN}$-associated deaths in the HIV or malignant neoplasm group) . This methodology has been previously applied in the assessment of mortality associated with the systemic connective tissue diseases [13]

AIRD autoimmune inflammatory rheumatic disease, $C I$ confidence interval, $C T D$ systemic connective tissue diseases, $H I V$ human immunodeficiency virus, $P M N$ all-cause pneumonia, $R A$ rheumatoid arthritis

\section{Reference}

13. Sartori Vieira C, de Rezende RPV, Mendes Klumb E, Cardoso Mocarzel LO, Altenburg Gismondi R (2019) Mortality profile related to the spectrum of systemic connective tissue diseases: a retrospective, population-based, case-control study. Lupus 28:1498-1500
Publisher's note Springer Nature remains neutral with regard to jurisdictional claims in published maps and institutional affiliations. 